Benchmarks

\title{
Hepatitis C genotype 1a replicon improved through introduction of fitness mutations
}

\author{
Christian Voitenleitner ${ }^{1}$, Jill Bechtel ${ }^{1}$, Ann Arfsten ${ }^{2}$, and Robert \\ Hamatake ${ }^{1}$ \\ ${ }^{1}$ GlaxoSmithKline, Research Triangle Park, NC, USA, ${ }^{2}$ InterMune, \\ Brisbane, CA, USA
}

BioTechniques 52:273-275(April 2012) doi 10.2144/000113841

Keywords: Hepatitis C; genotype 1a replicon; fitness; transient assay

The use of subgenomic replicon systems has long been a valuable screening tool for the discovery of small molecule antivirals against Hepatitis $\mathrm{C}$ virus. While genotype 1a replicon systems have been widely used in stable systems, use in transient assays has been hampered by low signal. Here we describe the generation of a more robust genotype $1 \mathrm{a}(\mathrm{H} 77)$ replicon through the introduction of two fitness mutations, NS4A-K1691R and NS4B-E1726G, for use in transient transfections. While these mutations significantly improved the signal to noise ratio, leading to more robust data, they have no effect on the potency of tool compounds against various targets of $\mathrm{HCV}$, thereby making this new system a powerful tool for screening of compounds against the genotype 1 a replicon.

Hepatitis $\mathrm{C}$ virus (HCV) is a plus-strand RNA virus in the Flaviviridae family with replication restricted to hepatocytes of humans and chimpanzees. HCV infects more than $3 \%$ of the world population, leading to increased risk of liver cirrhosis and hepatocellular carcinoma. The search for $\mathrm{HCV}$ model systems has been hampered by the fact that $\mathrm{HCV}$ isolates taken from patients replicate poorly, if at all, in in vitro cell culture systems. The first selectable subgenomic replicon capable of replicating autonomously-generated in the laboratory of Ralf Bartenschlager (1) - revealed a series of so called adaptive mutations that allow this construct to replicate in tissue culture (2-4). These subgenomic HCV replicons have proven to be powerful tools for studying viral replication or performing drug screening, and additional mutations have been reported that enhance the fitness of stable genotype la systems (5). Given the error prone nature of the RNA-dependent RNA polymerase NS5B, resistance to direct acting antivirals develops rapidly necessitating the ability to screen a compound against a panel of resistant replicons during drug development. Since keeping stable replicon cell lines of all these mutant replicon systems is prohibitive, mutant replicons are often used in transient assays rather than in stable cell lines. In transient assays, the hepatoma cell line Huh7-Lunet (6) is electroporated with in vitro transcribed HCV RNA that contains a luciferase reporter. The transfected cells are transferred onto 96-well plates containing compounds of interest in a dilution series. Luciferase activity is measured after 72 $\mathrm{h}$ to establish $\mathrm{EC}_{50}$ values of compounds against certain replicons (Figure 1A). The genotype 1a-H77 subgenomic replicon (7), which contains a luciferase/neomycin (neo) reporter cassette as well as the structural proteins NS3 through NS5B and two adaptive mutations in NS3/4A and NS5A, P1496L and S2204I, respectively, has very low luciferase levels after $72 \mathrm{~h}$ resulting in a low signal to noise ratio (Figure 2A).
This low signal makes it difficult to obtain robust data on the potency of compounds against the 1a-H77 replicons, especially those resistance mutant replicons exhibiting even further diminished replication capacity.

In order to quickly evaluate lead compounds against our large panel of resistance mutations, we needed to create a system with a quick luciferase reporter readout that had a robust signal to noise ratio at the assay readout time of $72 \mathrm{~h}$. An additional advantage of a transient system is that there is no additional accumulation of mutations as can happen in a stable cell line propagated for several cell division cycles. In order to achieve this goal, we started with a replicon system that originated from a resistance screen with an $\mathrm{HCV}$ inhibitor targeting NS4B and revealed a mutation in NS4B, E1726G, which conferred fitness to the $\mathrm{la}-\mathrm{H} 77$ replicon. Replicons carrying this mutation manifested higher luciferase units at the $72 \mathrm{~h}$ time point of a transient transfection (Figure 2A), yet did not impact the activity of $\mathrm{HCV}$ inhibitors to various targets (Figure 2C). Furthermore, a publication from Stan Lemon's laboratory reported several mutations that enhanced replication of the 1a-H77 replicon (8). We chose the mutation in the NS4A gene, K1691R, for minimal interference with our compound targets, while still displaying increased fitness in the 1a replicon. In an effort to further improve the luciferase signal resulting from transient transfection of the 1a-H77 replicon, we introduced both mutations into the 1a-H77 subgenomic replicon. Site-directed mutagenesis using the Quikchange Kit from Stratagene resulted in the 1a-fit (1a-H77-K1691R) E1726G) replicon (Figure 1B).

In order to assess and compare the robustness of these replicons, we performed a transient transfection, followed by a time course up to the assay readout time of 72 h (Figure 2A). After the initial burst of luminescence at $4 \mathrm{~h}$, which stems from translation of the luciferase gene from the transfected RNA rather than replicated RNA, we saw an initial decline in all samples, which indicated the lag until proteins from the replicated RNA begin to be made. At 48 h, 1a-E1726G showed higher signal than 1a-H77; this difference continued through the $72 \mathrm{~h}$ time point. The la-fit vector consistently showed the highest luminescence, with a 10 -fold higher readout than the 1a-E1726G and a hundred-fold higher readout than 1a-wt at $72 \mathrm{~h}$. Some mutations obtained from resistance screens and introduced into the replicon system are known to reduce replicon fitness significantly. One example 


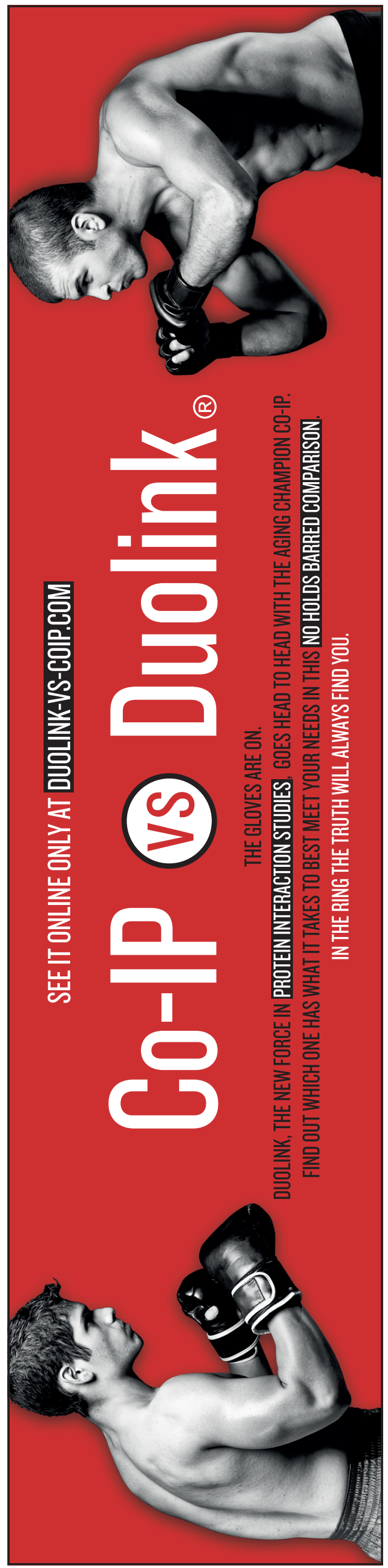

A

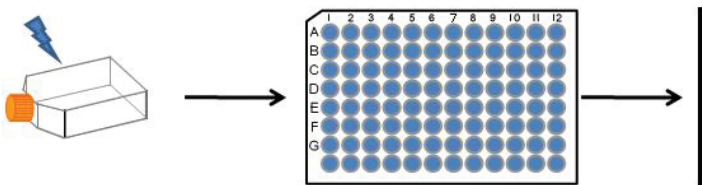

Electroporation of RNA into Huh-7-Lunet cells
Transfer of transfected cells onto plates containing compound

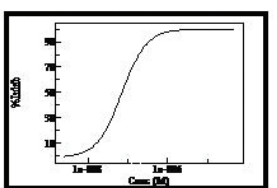

Luciferase assay to obtain IC50 values

\section{B}

1a-fit (1a-H77-K1691R/E1726G)

Figure 1. Schematic of transient transfections. (A) Transient transfection. Five $\mu g$ of in vitro translated RNA was electroporated into $1.25 \times 10^{7}$ Huh-7-Lunet cells suspended in $400 \mu \mathrm{L}$ cytomix (10) supplemented with $2 \mathrm{mM}$ ATP and $5 \mathrm{mM}$ glutathione. Cells were electroporated at $270 \mathrm{~V}, 950 \mu \mathrm{F}$ and infinite resistance and immediately transferred to a conical tube containing $40 \mathrm{~mL}$ of DMEM. $199 \mu \mathrm{L}$ of the cell suspension was plated in 96-well plates containing $1 \mu \mathrm{L}$ compound in DMSO at an appropriate concentration and dilution series across each row. Plates were incubated at $37^{\circ} \mathrm{C}$ and $5 \% \mathrm{CO}_{2}$ for 3 days. For the luciferase assay, the supernatant was replaced by $25 \mu \mathrm{L}$ BrightGlo reagent (Promega) and read in an Envision 2103 Multilabel reader. The $I C_{50}$ values were determined with appropriate programs. (B) The la-fit replicon consists of a luciferase/neomycin (Neo) reporter/selection cassette, preceded by the HCV5'-UTR and the nonstructural proteins from NS3 to NS5B. It also carries the H77 adaptive mutations P1496L in NS3/4A and S2204I in NS5A. Additionally, the vector contains two additional mutations, the K1691R mutation in NS4A and the E1726G mutation in NS4B.

A

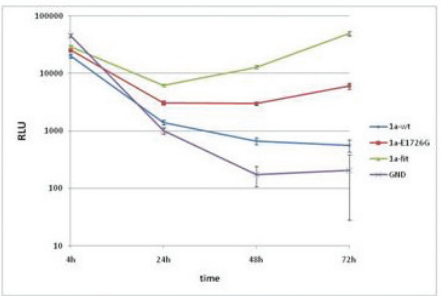

B

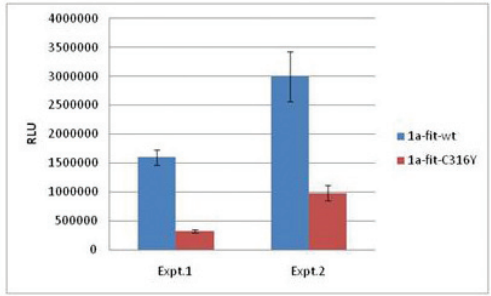

C

\begin{tabular}{|c|c|c|c|}
\hline Compound/Target & 1A-H77 EC 50 [nM] & 1a-E1726G [nM] & 1A-fit EC 50 $_{\text {[nM] }}$ \\
\hline BILN-2061/NS3/4A & $7.85 \pm 0.12$ & $6.21 \pm 0.86$ & $5.55 \pm 0.45$ \\
\hline NS4B & $6.67 \pm 1.29$ & $7.71 \pm 0.37$ & $7.31 \pm 0.40$ \\
\hline BMS-790052/NS5A & $0.03 \pm 0.002$ & $0.03 \pm 0.005$ & $0.02 \pm 0.001$ \\
\hline GS-9190/NS5B & $9.06 \pm 2.08$ & $12.73 \pm 2.11$ & $8.25 \pm 4.50$ \\
\hline HCV-796/NS5B & $6.03 \pm 0.93$ & $6.22 \pm 1.32$ & $6.83 \pm 0.88$ \\
\hline
\end{tabular}

Figure 2. The 1a-fit replicon shows enhanced fitness in transient assays. (A) Time-course of luminescence with 1a-wt, 1a-E1726G, 1a-fit and the replication deficient negative control GND. After a transient transfection with RNA of various vectors, Luciferase reads were taken after $4 \mathrm{~h}, 24 \mathrm{~h}, 48 \mathrm{~h}$ and $72 \mathrm{~h}$. The standard deviation of each time point is shown by error bars. The $P$ values of $1 \mathrm{a}-\mathrm{wt} / \mathrm{1a}-\mathrm{E} 1726 \mathrm{G}$ and of 1a-E1726G/1a-fit were significant with 3e-23 and 2e-27, respectively. (B) Transient transfection of Huh7-Lunet cells with either the 1a-fit or the 1a-fit-C316Y RNA. The luminescence of 2 independent experiments is shown in a linear scale in relative luciferase units. (C) Comparison of $\mathrm{EC}_{50}$ values of $1 \mathrm{a}$ $\mathrm{H} 77$ and $1 \mathrm{a}$-fit replicon systems with HCV inhibitors against different targets.

is the NS5B site IV mutation in 1a, C316Y, which has only $7 \%$ replication capacity compared with la-wildtype (9). We tested this mutant replicon and showed that, although replication fitness is still impaired relative to wildtype, the luciferase units of 1a-fit-C316Y after $72 \mathrm{~h}$ are high enough $(>300,000 \mathrm{RLU})$ to give a robust signal to noise ratio (Figure $2 \mathrm{~B}$ ). This signal represents a vast improvement when compared with the signal of $\mathrm{C} 316 \mathrm{Y}$ in the original 1a-H77, which ranged from about 1500 to 3000 RLU.

To verify that the two newly introduced mutations had no effect on compound potency of $\mathrm{HCV}$ inhibitors hitting various targets, we tested and compared a series of inhibitors against the $1 \mathrm{a}-\mathrm{H} 77$, the 1a-E1726G and the 1a-fit replicons (Figure 2C). When tested against a variety 
of compounds targeting protease, NS4B, NS5A, and various sites of NS5B, the $1 \mathrm{a}-$ fit replicon resulted in the same compound potency as the 1a-H77 or the 1a-E1726G replicon, suggesting that neither of these two newly introduced mutations altered compound potencies, but were merely conferring fitness to the replicon.

In summary, we have shown that introducing two mutations in NS4A and NS5B, respectively, could enhance replication fitness and increased the signal to noise ratio about 100 -fold compared with the $1 \mathrm{a}-\mathrm{H} 77$ replicon, resulting in more robust data for $\mathrm{HCV}$ inhibitors. This newly described replicon construct provides a quick reporter gene response through its Luciferase readout, as well as a robust signal for reliable determination of compound potencies. Because of the relative ease of introduction of mutations into the replicon, this system can be used to quickly profile a large number of compounds on a panel of resistance mutations against various $\mathrm{HCV}$ targets.

\section{Acknowledgments}

The authors would like to thank Ermias Woldu at GlaxoSmithKline for his technical assistance.

\section{Competing interests}

The authors declare no competing interests.

\section{References}

1. Lohmann, V., F. Korner, J. Koch, U. Herian, L. Theilmann, and R. Bartenschlager. 1999. Replication of subgenomic hepatitis $\mathrm{C}$ virus RNAs in a hepatoma cell line. Science 285:110113.

2. Krieger, N., V. Lohmann, and R. Bartenschlager. 2001. Enhancement of hepatitis C virus RNA replication by cell culture-adaptive mutations. J. Virol. 75:4614-4624.

3. Lohmann, V., F. Korner, A. Dobierzewska, and R. Bartenschlager. 2001. Mutations in hepatitis $C$ virus RNAs conferring cell culture adaptation. J. Virol. 75:1437-1449.

4. Lohmann, V., S. Hoffmann, U. Herian, F. Penin, and R. Bartenschlager. 2003. Viral and cellular determinants of hepatitis C virus RNA replication in cell culture. J. Virol. 77:3007-3019.

5. Robinson, M., H. Yang, S.C. Sun, B. Peng, Y. Tian, N. Pagratis, A.E. Greenstein, and W.E. Delaney. 2010. Novel hepatitis $C$ virus reporter replicon cell lines enable efficient antiviral screening against genotype 1a. Antimicrob. Agents Chemother. 54:3099-3106.

6. Blight, K.J., J.A. McKeating, and C.M. Rice. 2002. Highly permissive cell lines for subgenomic and genomic hepatitis $C$ virus RNA replication. J. Virol. 76:13001-13014.

7. Blight, K.J., J.A. McKeating, J. Marcotrigiano, and C.M. Rice. 2003. Efficient replication of hepatitis C virus genotype 1a RNAs in cell culture. J. Virol. 77:3181-3190.

8. Yi, M. and S.M. Lemon. 2004. Adaptive mutations producing efficient replication of genotype 1a hepatitis $\mathrm{C}$ virus RNA in normal Huh7 cells. J. Virol. 78:7904-7915.

9. McCown, M.F., S. Rajyaguru, S. Kular, N. Cammack, and I. Najera. 2009. GT-1a or GT-1b subtype-specific resistance profiles for hepatitis $C$ virus inhibitors telaprevir and HCV-796. Antimicrob. Agents Chemother. 53:2129-2132.

10. van den Hoff, M.J., A.F. Moorman, and W.H. Lamers. 1992. Electroporation in 'intracellular' buffer increases cell survival. Nucleic Acids Res. 20:2902.
Received 13 October 2011; accepted 08 February 2012

Address correspondence to Christian Voitenleitner, GlaxoSmithKline, 5 Moore Drive HCV DPU, N3.3235C, Research Triangle Park, NC, USA. Email: christian.a.voitenleitner@gsk. com

To purchase reprints of this article, contact: biotechniques@fosterprinting.com

\section{The Right Surface}

for your advanced tissue culture models

Tired of searching the right surfaces and culture conditions for your cells?

Try BD's surface selection guide: www.bdbiosciences.com/eu/surfaces

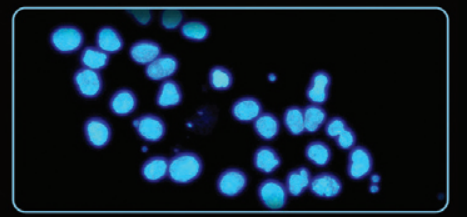

BD Falcon ${ }^{\text {m }}$

- tissue culture-treated

for almost all cell lines using traditional culture methods
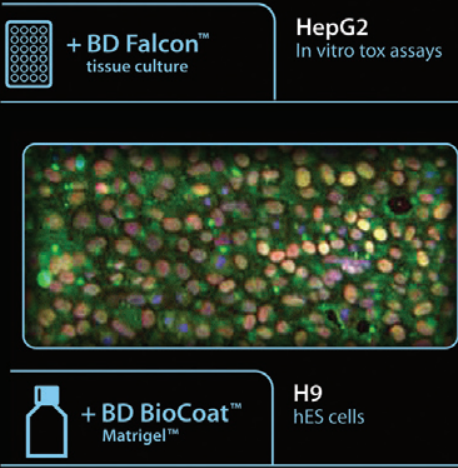

\section{BD BioCoat ${ }^{\mathrm{TM}}$}

- coated with extracellular matrices or PDL

- mimics the in vivo environment

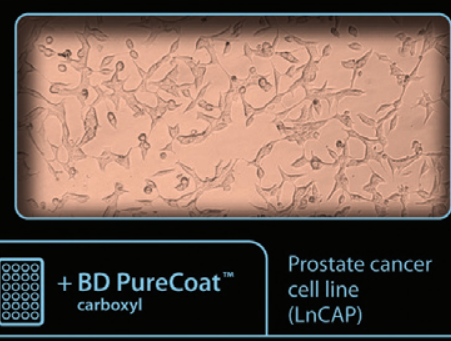

\section{BD PureCoat ${ }^{\text {TM }}$}

- animal-free and chemically defined

enhanced performance for fastidious cell types and advanced culture conditions (e.g. reduced serum/serum-free, transfected cells)

The

RIGHT SURFACE for every cell.

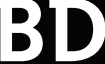

BD Biosciences bdbiosciences.com/eu Tel.: (+32) 24009895 Fax: (+32) 24017094 help.biosciences@europe.bd.com 